\title{
A New Method to Estimate the Real Upper Limit of the False Alarm Rate in a 3 Accelerometry-Based Fall Detector for the Elderly
}

\author{
Cristina Soaz ${ }^{1}$, Christian Lederer ${ }^{2}$ and Martin Daumer ${ }^{3}$
}

\begin{abstract}
Falls are a major concern for the elderly and their ability to remain healthy. Fall detection systems may notify emergency responders when no one apart from the injured is present. However, their real-world application is limited by a number of factors such as high false positive rates, lowcompliance, poor-usability and short battery lifetime. In order to improve these aspects we have developed a miniaturized 3D accelerometer integrated in a belt buckle, the actibelt ${ }^{\circledR}$, and a fall detection algorithm. We have used a new evaluation method to assess the upper limit of the false alarm rate of our algorithm using a large set of long term standardized acceleration measurements recorded under real life conditions. Our algorithm has a false alarm rate of seventeen false alarms per month and has the potential to be reduced down to at most three false alarms per month when activities which require the sensor to be removed are eliminated. In laboratory settings, the algorithm has a sensitivity of $100 \%$. The algorithm was sucessfully validated using data from a real-world fall.
\end{abstract}

\section{INTRODUCTION}

Health care among the elderly is a rising social and economic concern as life expectancy trends grow. It is becoming increasely clear for commissioners and decision makers that new care models are needed for the sustainability of modern society. The European Commission has recently identified a "need for urgent action to shift the focus from acute, reactive, and hospital-based care for the elderly to long term, proactive and home-based care" [1].

A major problem is that falls in older people are frequent and many of the elderly who fall at home become helpless and require assistance to get up. Tinetti and colleagues [2] conducted a study with 313 noninjured fallers living in the community, aged 72 years and older, of which $47 \%$ reported inability to get up after at least one fall. Fleming et al. [3] found that the $80 \%(53 / 66)$ of the elderly fallers in their 90's were unable to get up after a fall, and $30 \%$ had lain on the floor for an hour or more. Of those who were alone when they fell, $80 \%$ did not activate their alarms. In this context, user-friendly automatic or semiautomatic emergency response and monitoring systems can extend the length of time that seniors are able to live in their own homes.

The focus of this paper lies in a critical part of such alarm system: the sensitivity and false alarm rate of the fall

\footnotetext{
*This work was not supported by any external organization

${ }^{1}$ C. Soaz is with the SLCMSR e.V. - The Human Motion Institute, Munich, Germany, and with the Faculty of Electrical Engineering and Information Technology, TUM, Munich, Germany soaz at slcmsr.org, soaz at rcs.ei.tum.de

${ }^{2}$ C. Lederer is with the SLCMSR e.V. - The Human Motion Institute, Munich, Germany lederer at slcmsr.org

${ }^{3}$ M. Daumer is with the SLCMSR e.V. - The Human Motion Institute, Munich, Germany, and the Trium Analysis Online GmbH, Munich, Germany daumer at slcmsr.org
}

detection algorithm. Together with the usability aspect of the activity monitor these are known to be the key success factors for fall detection systems. To satisfy the condition that the algorithm runs in real-time for an extended period of time in the microcontroller of the monitoring device and to guarantee a high user acceptance, a low power activity monitor integrated in a belt buckle, the actibelt ${ }^{\circledR}$ [4]-[6], is used as key part of the study.

\section{METHODS}

\section{A. Acceleration sensor}

The actibelt (Fig. 1) is a custom-built 3D accelerometer (ADXL 345 BCCZ Analog Devices), with high resolution (13-bit) measurement up to $\pm 6 \mathrm{~g}(\mathrm{~g}=9.81 \mathrm{~m} / \mathrm{s} / \mathrm{s})$ and $100 \mathrm{~Hz}$ sample frequency. The sensor is integrated in a belt buckle placed near the center of mass of the human body and it records the accelerations relative to the trunk along the vertical, mediolateral and anterioposterior directions.

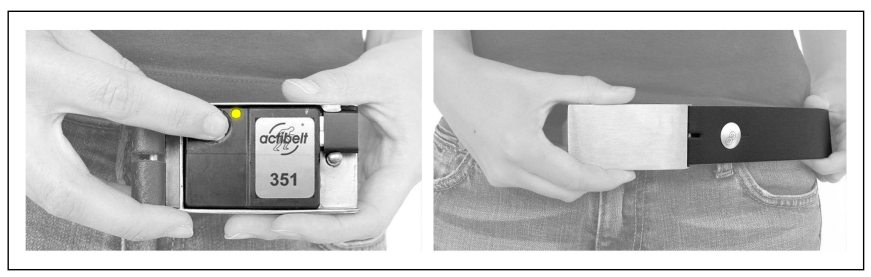

Fig. 1. Photos of the actibelt ${ }^{\circledR}$ accelerometer used for the data acquisition

It measures the static acceleration of gravity as well as dynamic acceleration resulting from motion or shock. Its high resolution $(4 \mathrm{mg} / \mathrm{LSB})$ enables resolution of inclination changes of as little as $0.25^{\circ}$. The unit is waterproof. The dimensions are $65 \times 38 \times 9 \mathrm{~mm}$ and it weighs $34 \mathrm{gr}$. The $512 \mathrm{MB}$ storage capacity permits and autonomous operating time of about 10 days. The power supply is provided by a rechargeable LiPo battery that lasts approximately one month and can be recharged via USB in 2 hours.

The actibelt ${ }^{\circledR}$ (version actibelt ${ }^{\circledR}$-BLU) includes a temperature and barometric pressure sensor (Bosch BMP085), a bluetooth ${ }^{\complement}$ module (LMX9838 National Semiconductors) and a $125 \mathrm{KHz}$ RFID module. The wireless device is equipped with a micro USB port and a removable $8 \mathrm{~GB}$ micro SD card.

\section{B. Subject Database}

1) Historical Database: During the last 6 years, the Sylvia Lawry Center for Multiple Sclerosis Research has 
built a database with more than 100,000 hours of accelerometry recordings. The standardized placement of the accelerometer as well as unchanged sensor type and sampling frequency allowed to build a valuable resource for algorithm development and refinement. For this study and to validate the upper limit of the realistic false alarm rate of our algorithm we selected 3 subsamples from the database according to age criteria: adolescents, middle-age and elderly people. Despite the fact that the fall detection system is thought to be used among the elderly we designed our study to include subjects of a wide age range to confirm our assumption about the potential decrease of false alarm rate with age.

In total, 2,415.9 hours (100.7 days) of continuous weekly acceleration measurements corresponding to ADLs (Activities of Daily Living) in free living conditions were used for the validation. The subsamples are distributed as follows: the first cohort is comprised of 3 diabetic adolescents (10.5yr), the second cohort is formed by 6 healthy individuals ( 6 male, $32.3 \mathrm{yrs}$ ) and the third one by 5 elderly women (70.2 yrs) diagnosed with osteoporosis but without mobility limitations.

2) Prospective Data Collection: For the development and improvement of the fall detection algorithm a series of fall-like activities and simulated fall events (Table I) were performed by two healthy individuals ( 2 male; age: 46 and 79 yrs; height: 1.84 and $1.74 \mathrm{~m}$ ) in a controlled laboratory setting at the Sylvia Lawry Center for Multiple Sclerosis Research (Munich, Germany). Both subjects were informed about the aims of the activity measurement and had given written informed consent.

3) Real-world fall: During the course of this research, a patient (male; 56 yrs) who participated in a rehabilitation exercise therapy fell accidentally out of the clinic while being monitored with the actibelt ${ }^{\circledR}$. The fall resulted in a fracture of the neck of femur. This unfortunate accident provided us with an extremely valuable data for two main reasons: 1) the difficulty that in itself entails to get a real fall. As an example, to capture 100 real-world falls it would be necessary to record approximately 100,000 days of physical activity (300 person years) [8]. And 2), the detailed documentation about the real-world fall and previous weeks; in addition to the selfreported information, we have 24-hour actibelt ${ }^{\circledR}$ recordings and clinical functional test measurements (Timed Up and Go test, 10 meter test and 6 minute walking test) from the two weeks prior to the accident.

\section{Trial protocol}

The trial protocol consists of a series of 16 real falls in laboratory conditions and 7 fall-like activities which should not trigger an alarm. The experiments were performed on a sofa and on a crash pad $8 \mathrm{~cm}$ thick (see Table I). Falls were performed in three directions: backwards, forwards and sideways, starting from a walking and a standing position and were selected to mimic typical real world situations. E.g., sequence 17 and 18 reflect situations where the subject stumbles before falling down. Sequence 12, 13 and 14 imitate the everyday scenario of a person lying down on the bed and, occasionally, tossing and turning. The last task (number 23) simulates someone who sits down and loses consciousness resulting in a fall.

All participants performed the sequence once and were instructed about how to execute the specific task at the beginning of each activity. All activities start with a pause of five seconds, followed by a tap on the belt and other fivesecond pause. All activities were video recorded. One faller was the senior author of this manuscript.

\section{FALL DETECTION ALGORITHM}

\section{A. Feature extraction}

The preprocessing of the raw data was performed according the filtering methods described by Karantonis et al. in [7]. First, the acceleration raw data is denoised using a median filter $(n=3)$. Then, in order to get the gravity acceleration component (GA) the output is low pass filtered, whereby a custom third-order elliptical infinite impulse response (IIR) filter with cut-off frequency at $0.25 \mathrm{~Hz}$. The acceleration forces originated by the body movements (BA) are extracted by subtracting the gravity acceleration to the denoised signal. After that, both components, GA and BA, are transformed into spherical coordinates.

By comparing the magnitude of the acceleration vector produced by the body movement, $\rho_{B A}$, with a certain threshold we determine a possible impact against the ground. This magnitude can be calculated as following:

$$
\rho_{B A}[i]=\sqrt{x^{2}[i]+y^{2}[i]+z^{2}[i]}
$$

where $x[i]$ is the $i^{t h}$ sample along the $x$ axis (likewise for $y[i]$ and $z[i])$.

The $\theta$ coordinate of the gravitational component, $\theta_{G A}$, is used to differentiate between standing/sitting position and lying orientation. The value $\theta_{G A}$ (equation 2 ) estimates the angle formed by the vertical axis of the upper body and the ground.

$$
\theta_{G A[i]}=\cos ^{-1}\left(z[i] / \rho_{B A}[i]\right)
$$

where $z[i]$ is the $i^{\text {th }}$ sample along the vertical axis.

To distinguish between rest and activity phases the sum of the windowed standard deviation, SWSD (equation 3), of the $\rho_{B A}$ signal was calculated for a window length $L=50$ samples.

$$
S W S D=\sum_{i=1}^{n} \sigma_{i}
$$

where

$$
\sigma_{i}=\sqrt{\frac{\sum_{j=(i-1) L}^{i L-1}\left(\rho_{B A}[j]-\bar{\rho}_{B A_{i}}\right)^{2}}{L}}
$$

with

$$
\bar{\rho}_{B A_{i}}=\frac{\sum_{j=(i-1) L}^{i L-1} \rho_{B A}[j]}{L}
$$


TABLE I

TEST PROTOCOL

\begin{tabular}{|c|c|c|c|c|}
\hline Surface & Starting position & $\mathbf{N}^{\circ}$ & Instructions $*$ & Desired outcome \\
\hline \multirow{18}{*}{ Crash pad } & \multirow{4}{*}{$\begin{array}{l}3 \text { steps away from the } \\
\text { crash pad }\end{array}$} & 1 & walk, fall forwards softly, stay in lying position for $10 \mathrm{sec}$ & alarm \\
\hline & & 2 & walk, fall forwards softly, writhe softly for $10 \mathrm{sec}$ & alarm \\
\hline & & 3 & walk, fall forwards hardly, stay in lying position for $10 \mathrm{sec}$ & alarm \\
\hline & & 4 & walk, fall forwards hardly, writhe softly for $10 \mathrm{sec}$ & alarm \\
\hline & \multirow{4}{*}{$\begin{array}{l}1 \text { foot away from the } \\
\text { crash pad }\end{array}$} & 5 & fall forwards softly, stay in lying position for $10 \mathrm{sec}$ & alarm \\
\hline & & 6 & fall forwards softly, turn on your back, stay in lying position for $10 \mathrm{sec}$ & alarm \\
\hline & & 7 & fall forwards hardly, stay in lying position for $10 \mathrm{sec}$ & alarm \\
\hline & & 8 & fall forwards hardly, turn on your back, stay in lying position for $10 \mathrm{sec}$ & alarm \\
\hline & \multirow{8}{*}{$\begin{array}{l}2-3 \text { feet away from the } \\
\text { crash pad }\end{array}$} & 9 & fall backwards softly, stay in lying position for $10 \mathrm{sec}$ & alarm \\
\hline & & 10 & fall backwards hardly, turn on your back, stay in lying position for $10 \mathrm{sec}$ & alarm \\
\hline & & 11 & fall backwards softly, get up after $5 \mathrm{sec}$ & no alarm \\
\hline & & 12 & lie down backwards, stay in lying position for $10 \mathrm{sec}$ & no alarm \\
\hline & & 13 & lie down backwards, writhe heavily for $10 \mathrm{sec}$ & no alarm \\
\hline & & 14 & lie down backwards, writhe heavily for $10 \mathrm{sec}$ and tap on the belt & no alarm \\
\hline & & 15 & fall softly sideways, stay in lying position for $10 \mathrm{sec}$ & alarm \\
\hline & & 16 & fall hardly sideways, stay in lying position for $10 \mathrm{sec}$ & alarm \\
\hline & \multirow{2}{*}{$\begin{array}{l}1 \text { foot and } 3 \text { steps away } \\
\text { from the crash pad }\end{array}$} & 17 & $\begin{array}{l}\text { walk, stumble after } 3 \text { steps and fall forwards softly, stay in lying position for } \\
10 \mathrm{sec}\end{array}$ & alarm \\
\hline & & 18 & $\begin{array}{l}\text { walk, stumble after } 3 \text { steps and fall forwards hardly, stay in lying position for } \\
10 \mathrm{sec}\end{array}$ & alarm \\
\hline \multirow{4}{*}{ Sofa } & \multirow{5}{*}{$\begin{array}{l}\text { half a foot away from } \\
\text { the sofa, facing it } \\
\text { backwards }\end{array}$} & 19 & fall on the sofa, leave your upper body in an upright position, stay for $10 \mathrm{sec}$ & no alarm \\
\hline & & 20 & $\begin{array}{l}\text { fall on the sofa, leave your upper body in an upright position, writhe softly, } \\
\text { stay for } 10 \mathrm{sec}\end{array}$ & no alarm \\
\hline & & 21 & sit on the sofa, change into a lying position, stay for $10 \mathrm{sec}$ & no alarm \\
\hline & & 22 & fall on the sofa, change into a lying position, stay for $10 \mathrm{sec}$ & no alarm \\
\hline $\begin{array}{l}\text { Sofa and } \\
\text { crash pad }\end{array}$ & & 23 & $\begin{array}{l}\text { sit on the sofa, wait } 5 \text { seconds, tap on the belt buckle, wait } 10 \mathrm{sec} \text {, fall forwards } \\
\text { on the crash pad, stay in lying position for } 10 \mathrm{sec}\end{array}$ & alarm \\
\hline
\end{tabular}

*Explanation: fall softy: break the fall with your arms and knees, writhe softly: $10^{\circ}-15^{\circ}$ change of position, writhe heavily: approx. $90^{\circ}$ change of position, stumble: after 3 steps, hook your one leg behind the other and fall.

\section{B. Threshold calculation}

To find the optimal thresholds for the identification of the fall impacts and the relative body position, the controlled fall data set (30 simulated falls; Table I) was divided randomly in two equal groups, one selected as the training group. The thresholds were calculated using only data from this dataset.

The threshold for $\rho_{B A}$ was chosen such that we achieve $100 \%$ sensitivity when the algorithm is run over the training data and it was selected as the minimum rounded down value of the acceleration peaks produced by the fall impacts in the signal $\rho_{B A}$. This number, $t h_{m a g}$, is equal to $1.9 \mathrm{~g}$. The tilt angle threshold, $t h_{\text {ang }}$, was selected as the minimum average value of $\theta_{G A}$ during the next 13 seconds after the fall, and its value is 49.8 degrees. The three seconds immediately after the fall were ignored due to potential residual movement relating to the knock into the crash pad. To identify periods of inactivity the SWSD of the magnitude vector signal should be under the threshold, $t h_{a c t}=3.0 \mathrm{~g}$.

\section{Fall detection algorithm}

The fall detection algorithm is based on the detection of an impact by the comparison of the magnitude of the acceleration vector produced by the body movement to a preset threshold and on the body orientation after the impact.

The $\rho_{B A}$ samples which surpass $t h_{m a g}$ are grouped together in a block provided that the sample difference in between them is not more than 15 samples and the length of the block is maximum 1 second duration (100 samples). Each of these blocks is classified as a potential fall. In a second step, the relative position of the trunk in respect of the perpendicular vector to the ground's plane is calculated along the 10 seconds interval within the 3rd and 13th second after the first sample of the block. It has been empirically found that an interval of 3 seconds after the fall is enough for the tilt signal to stabilize in case of a fall occurs. If the trunk tilt given by $\theta_{G A}$ within this period goes over the threshold $t h_{a n g}$, the person is considered to be in a nonstanding position. An alarm is then only activated if the person was in a standing position before the impact and the quantity of movement after it does not go over the threshold $t h_{\text {act }}$.

\section{Validation method}

In order to evaluate the performance of our fall detector, the algorithm was ran, first, over the whole test battery of activities and simulated fall events (Table I); second, over the continuous acceleration data set of ADLs recorded in a free-living environment and then over the real fall data.

The output of the first analysis yields an estimation for the sensitivity of the algorithm on the basis that the fall scenarios covered in the experiment protocol are highly representative of a real fall, whereas the free-living activity recordings give an upper limit for the false alarm rate (it is unknown whether some real alarms were within the ones detected as such) in an entirely realistic scenario. Finally, the real-world fall was useful to test the ecological validity of our test protocol. The fall-like activities included in the protocol were intended to be used for the refinement of the algorithm or in event 
of troubleshooting in case that the false alarm rate were much higher than the expected one but never to serve as an estimation for the specificity. The ecological validity of the specificity calculated as such is void due to the absence of a prevalence value.

\section{RESULTS}

In total, 2,416 hours of uncontrolled ADL activities, 46 simulated falls and fall-like events as well as one realworld fall were analyzed in $\mathrm{R}$ retrospectively. The overall sensitivity measures the percentage of positives (alarms) in the prospective data collection which are correctly identified as such. In our study we obtained a sensitivity equal to $100 \%$.

The algorithm detected a fall-like impact (see Fig.2) when ran over the file which contained the real-world fall at around the same time when it was reported that it happened. 1,270 100 samples per axis $\times 3$ axis (approx. 3,5 hours recording) were analyzed and the results did not show any false alarm.

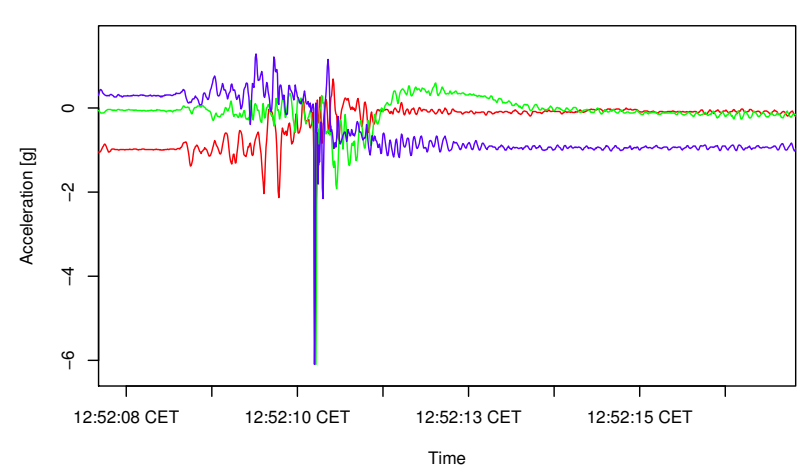

Fig. 2. Real fall detected by the algorithm. Red line is vertical axis, green is mediolateral axis and blue is anterioposterior axis $(\mathrm{g}=9.81 \mathrm{~m} / \mathrm{s} / \mathrm{s})$

The upper limit for the false alarm rate was calculated separately for each cohort in a two-step evaluation. The results are shown in Table II. The UL-cure refers to the results obtained after eliminating the false positives due to the act of removing the device from the belt. Our assumption about false alarm rate decreases with age was confirmed.

TABLE II

UL-RAW/UL-CURE: UPPER LIMIT FALSE ALARM RATE WITHOUT/WITH DATA CURATION (FALLS PER MONTHS)

\begin{tabular}{|l||c||c||c||c|}
\hline & Mean age (yrs) & Recorded hours & UL-raw & UL-cure \\
\hline Cohort 1 & 10.5 & 688.1 & 135 & 127.7 \\
Cohort 2 & 32.3 & 732.1 & 26.6 & 11.8 \\
Cohort 3 & 70.2 & 995.7 & 17.4 & 2.9 \\
\hline
\end{tabular}

\section{CONCLUSION}

We have developed a promising methodology to detect falls using a body worn sensor, that has high sensitivity and an acceptable rate of false alarms. We think that it is a particular strength of the current study to have provided a strict upper limit for the realistic false alarm rate using historical accelerometry data. One should note that the specificity of an algorithm using fall data generated in laboratory settings is meaningless due to the absence of a prevalence value although it is still widely used as a measure for algorithm performance.

The success detecting a real fall evidence that the simulation protocol we chose for the algorithm development covered at minimum one real fall scenario. The lack of more and various types of real fallings restricts the ecologic validity of our result of $100 \%$ sensitivity. An open accelerometry database including real world-falls would be of considerable importance for a continuous and independent refinement and validation of fall detection and prevention algorithms - see also [8].

Future work should focus on the optimal implementation of the algorithm in the microcontroller of the actibelt ${ }^{\circledR}$ and aim at further decreasing the number of false alarms by sensor fusion and suppression mechanisms, e.g., through the use of smart phones and automatic ways of detecting sensor removal.

\section{ACKNOWLEDGMENT}

The authors would like to thank Karl Daumer, Ursula Muesch and Alexandra Iovkova for their help with data acquisition. We also would like to thank Julia Endres and the Neurorehabilitation Center Fries (Munich, Germany) for providing us with the real fall data.

\section{REFERENCES}

[1] Steering Group of the European Innovation Partnership on active and healthy ageing. "Strategic Implementation Plan For The European Innovation Partnership On Active And Healthy Ageing". Internet: http://ec.europa.eu/research/innovation-union/ index_en.cfm?section=active-healthy-ageing\&pg= implementation-plan Nov. 17, 2011 [March 2, 2012]

[2] M.E. Tinetti , W.L. Liu and E.B.Claus. Predictors and prognosis of inability to get up after falls among elderly persons. JAMA 1993;269:65-70

[3] J. Fleming, C.Brayne and Campbell. Inability to Get up after Falling, Subsequent Time on Floor, and Summoning Help: Prospective Cohort Study in People over 90. BMJ: British Medical Journal Vol. 337, No. 7681 (Nov. 29, 2008), pp. 1279-1282

[4] M. Daumer, K. Thaler, E. Kruis, W. Feneberg, G. Staude, and M. Scholz. Steps towards a miniaturized, robust and autonomous measurement device for the long-term monitoring of the activity of patients - ActiBelt. Biomedizinische Technik/Biomedical Engineering. 2007; 52: 149-155.

[5] M. Schimpl, C. Lederer and M. Daumer. Development and Validation of a New Method to Measure Walking Speed in Free-Living Environments Using the Actibelt ${ }^{\circledR}$ Platform. PLoS ONE 6(8): e23080. doi:10.1371/journal.pone.0023080

[6] M. Schimpl, C. Moore, C. Lederer, A. Neuhaus, J. Sambrook, et al. 2011 Association between Walking Speed and Age in Healthy, FreeLiving Individuals Using Mobile Accelerometry-A Cross-Sectional Study. PLoS ONE 6(8): e23299. doi:10.1371/journal.pone.0023299

[7] D. M. Karantonis, M. R. Narayanan, M. Mathie, N. H. Lovell, and B. G. Celler, "Implementation of a real-time human movement classifier using a triaxial accelerometer for ambulatory monitoring," IEEE Trans Inf Technol Biomed, vol. 10, pp. 156-67, 2006.

[8] F. Bagala, C. Becker, A. Cappello, L. Chiari, K. Aminian, et al. (2012) Evaluation of Accelerometer-Based Fall Detection Algorithms on Real-World Falls. PLoS ONE 7(5): e37062. doi:10.1371/journal.pone.0037062 